\title{
Устойчивость пространственно-временных солитонов в многомодовых волокнах
}

\author{
О.В. Штырина ${ }^{1,2}$, И.С. Чеховской ${ }^{1,2, *}$, Ю.С. Кившарь ${ }^{3}$, \\ С.К. Турицын ${ }^{1,4}$, И.А. Яруткина ${ }^{1,2}$, М.П. Федорук ${ }^{1,2}$ \\ ${ }^{1}$ Новосибирский государственный университет \\ ${ }^{2}$ Институт вычислительных технологий СО РАН \\ ${ }^{3}$ Австралийский Наџиональный Университет, Канберра, Австралия \\ ${ }^{4}$ Астонский Университет, Бирмингем, Великобритания \\ "E-mail: i.s.chekhovskoy@gmail.com
}

DOI:10.31868/RFL2018.114-115

Распространение импульсов в многомодовых волокнах с градиентным индексом (ММФ) описывается в параксиальном приближении стандартным нелинейным уравнением Шредингера (NLSE), полученным для медленно меняющейся огибающей импульса (включающей все моды) [1]:

$$
i \frac{\partial \psi}{\partial Z}=\frac{\beta_{2}}{2} \frac{\partial^{2} \psi}{\partial T^{2}}-\frac{1}{2 k_{0}}\left(\frac{\partial^{2} \psi}{\partial x^{\prime 2}}+\frac{\partial^{2} \psi}{\partial y^{\prime 2}}\right)+U\left(x^{\prime}, y^{\prime}\right) \psi-\gamma|\psi|^{2} \psi
$$

где $k_{0}$ - волновое число центральной частоты $\omega_{0}, \beta_{2}$ - дисперсия групповых скоростей и $\gamma$ - нелинейность, $\psi$ - медленно меняющаяся огибающая на центральной частоте $\omega_{0}$ от времени $T$ в системе отсчета, движущейся с групповой скоростью импульса. Рассмотрим эффективный потенциал $U\left(x^{\prime}, y^{\prime}\right)=$ $\left(k_{0} \Delta / R^{2}\right)\left(x^{2}+y^{2}\right)$, где $\Delta$ - разность индексов сердцевины и оболочки, а $R$ - радиус сердцевины волокна. Рассмотрим направляющую среду в случае $\Delta>0$.

Уравнение (1) имеет гамильтонову структуру и может быть переписано в безразмерном виде путем замены переменных, $\psi=\sqrt{P_{\text {norm }}} A_{s}, \quad T=T_{0} t$, $\left(x^{\prime}, y^{\prime}\right)=r_{0}(x, y), Z=Z_{0} z$ и $\mu=2 \Delta k_{0}{ }^{2} r_{0}{ }^{4} / R^{2}\left(\right.$ с $\left.\left(\gamma P_{\text {norm }}\right)^{-1}=T_{0}{ }^{2} / \beta_{2} \mid=k_{0} r_{0}{ }^{2}=Z_{0}\right)$ :

$$
i \frac{\partial A}{\partial z}=\frac{\delta H}{\delta A^{*}}=-\frac{\sigma}{2} \frac{\partial^{2} A}{\partial t^{2}}-\frac{1}{2}\left(\frac{\partial^{2} A}{\partial x^{2}}+\frac{\partial^{2} A}{\partial y^{2}}\right)+\frac{\mu}{2}\left(x^{2}+y^{2}\right) A-|A|^{2} A
$$

где $\sigma=\operatorname{sign}\left(\beta_{2}\right)= \pm 1$ (соответствует аномальной или нормальной дисперсии), а гамильтониан задается выражением:

$$
H=\left(\sigma I_{t}+I_{x}+I_{y}+\mu I_{3}-I_{4}\right)=\int d x d y d t\left[\sigma\left|A_{t}\right|^{2}+\left|A_{x}\right|^{2}+\left|A_{y}\right|^{2}+\mu\left(x^{2}+y^{2}\right)|A|^{2}-|A|^{4}\right]
$$

Существования солитонных решений недостаточно для анализа динамики нелинейных систем. Критической проблемой является устойчивость этих стационарных решений относительно различных возмущений. В 2- и 3-мерных NLSE солитоны неустойчивы, и любое начальное распределение с достаточно высокой мощностью $P$ коллапсирует к сингулярности [2,3]. В гамильтоновых системах, когда гамильтониан в рассматриваемой модели ограничен и решение соответствует его минимуму, соответствующий солитон является аттрактором. Если гамильтониан неограничен, то солитонные решения соответствуют седловым точкам гамильтониана и неустойчивы, как, например, в 3-мерном NLSE. В этом случае стационарного состояния не существует, и начальный волновой пакет либо диспергирует, что эффективно демонстрирует линейная динамика, либо начальное распределение поля коллапсирует, что математически соответствует образованию сингулярности поля.

Применяя стандартный вариационный подход [2,3] и следуя предыдущим исследованиям [1], мы анализируем устойчивость солитонов. Подход 
основывается на вариационном представлении (2) и аппроксимации решения $A(z, t, x, y)$ с помощью некоторой пробной функции. В качестве пробной функции выберем Гауссову, энергию которой будем обозначать $P$,

$$
A(x, y, t, z)=\frac{\sqrt{P_{0}}}{a(z) b^{1 / 2}(z)} \exp \left[-\frac{x^{2}+y^{2}}{2 a^{2}(z)}-\frac{t^{2}}{2 b^{2}(z)}\right] \times \exp \left[-i a(z)\left(x^{2}+y^{2}\right)-i \beta(z) t^{2}\right] .
$$

Гамильтониан теперь представим как функционал от масштабируемых параметров $a$ и $b: H(a, b)=\frac{P_{0} \pi^{3 / 2}}{2}\left(\frac{\sigma}{2 b^{2}}+\frac{1}{a^{2}}+\mu a^{2}-\frac{P_{0}}{2 \sqrt{2} a^{2} b}\right)$

Устойчивым солитонам при $\sigma=1$ соответствуют минимумы гамильтониана $H(a, b)$. Простой анализ экстремумов $H$ приводит к следующему условию существования локальных минимумов $H$ :

$$
\left(P_{0} / 4\right)^{4}<1 / 27 \mu \text {. }
$$
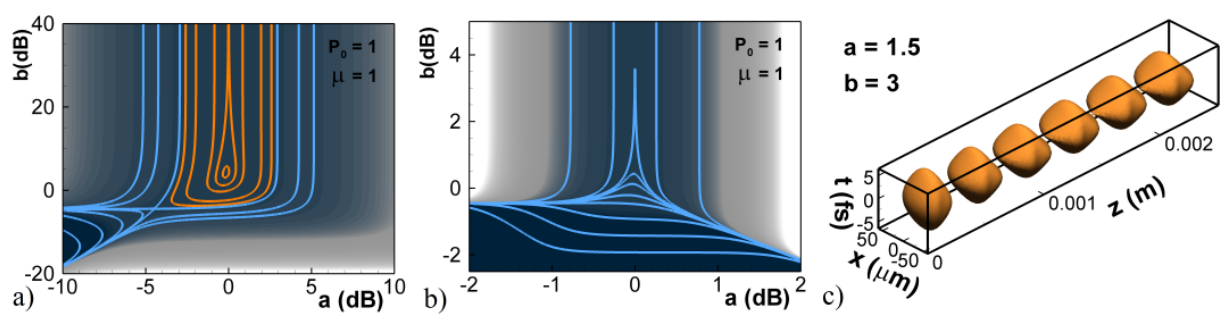

Рис.1. Гамильтониан $H(a, b)$ для $\mu=1$ и $P_{0}=1$. Здесь $\sigma=1$ (а) и $\sigma=-1$ (b). На (а) оранжевые кривые соответствуют траекториям устойчивой солитоноподобной динамики входного гауссовского импульса. (c) Результаты численного моделирования: изоповерхности $|A|^{2}(x, 0, t, z)$ представляют собой режим световой пули с солитонной динамикой в аномальном ММФ.

Рис. 1а показывает появление локальных минимумов при выполнении критерия существования. Чтобы описать параметры гауссовского импульса из оранжевой области, определим координаты седлового экстремума:

$$
a_{s}^{2}=\frac{2}{\sqrt{3 \mu}} \cos \left(\frac{\varphi}{3}-\frac{2 \pi}{3}\right), b_{s}=\frac{2 \sqrt{2}}{P_{0}} a_{s}^{2},
$$

где $\cos (\varphi)=-\sqrt{27 \mu}\left(\frac{P_{0}}{4}\right)^{2}$. Если выполнено условие (3), то область устойчивой солитонной динамики может быть определена следующим образом:

$$
a>a_{s} \text { и } b>b_{s} \text { и } H(a, b)<H\left(a_{s}, b_{s}\right) \text {. }
$$

Рис. 1с демонстрирует типичную солитоноподобную динамику импульса, который удовлетворяет условиям (4). Наблюдаются колебания начального гауссового импульса вблизи основной моды. Вариационный анализ подтверждает, что в нормальном дисперсионном волноводе (при $\sigma=-1$ ) устойчивых солитонных структур не существует. Рис. $1 \mathrm{~b}$ показывает отсутствие минимума Гамильтона. Существование локального экстремума по параметру $a$ при выполнении (4) подтверждает периодическую динамику пространственной длительности сигнала, период которой определяется длиной дифракции. Работа была выполнена при поддержке Российского научного фонда (проект 17-7230006).

\section{Литература}

[1] S. Raghavan, G. P. Agrawal, Opt. Commun. 180, 377-382 (2000).

[2] V.E. Zakharov, E.A. Kuznetsov, Phys. Usp. 5, 535-556 (2012).

[3] E.A. Kuznetsov, "Wave collapse in nonlinear optics," In Self- focusing: Past and Present. Topics in Applied Physics 114, (Springer, 2009), pp. 175-190. 